PROCEEDINGS OF THE

AMERICAN MATHEMATICAL SOCIETY

Volume 137, Number 3, March 2009, Pages 1161-1168

S 0002-9939(08)09666-4

Article electronically published on October 20, 2008

\title{
ON THE SAME $N$-TYPE CONJECTURE FOR THE SUSPENSION OF THE INFINITE COMPLEX PROJECTIVE SPACE
}

\author{
DAE-WOONG LEE
}

(Communicated by Paul Goerss)

\begin{abstract}
Let $\left[\varphi_{i_{k}},\left[\varphi_{i_{k-1}}, \cdots,\left[\varphi_{i_{1}}, \varphi_{i_{2}}\right], \cdots\right]\right]$ be an iterated commutator of self-maps $\varphi_{i_{j}}$ on the suspension of the infinite complex projective space. In this paper, we produce useful self-maps of the form $I+\left[\varphi_{i_{k}},\left[\varphi_{i_{k-1}}, \cdots\right.\right.$, $\left.\left.\left[\varphi_{i_{1}}, \varphi_{i_{2}}\right], \cdots\right]\right]$, where + means the addition of maps on the suspension structure of $\Sigma \mathbb{C} P^{\infty}$. We then give the answer to the conjecture saying that the set of all the same homotopy $n$-types of the suspension of the infinite complex projective space is the one element set consisting of a single homotopy type.
\end{abstract}

\section{INTRODUCTION}

Let $X^{(n)}$ be the $n$th Postnikov approximation of a space $X$. We recall that two $C W$-spaces $X$ and $Y$ are said to have the same $n$-type if the $n$th Postnikov approximations $X^{(n)}$ and $Y^{(n)}$ are homotopy equivalent. In the early years of algebraic topology, there was an important question posed by J. H. C. Whitehead: if $X$ and $Y$ are two spaces whose Postnikov approximations, $X^{(n)}$ and $Y^{(n)}$, are homotopy equivalent for each integer $n$, then does it follow that $X$ and $Y$ have the same homotopy type? It is well known that if $X$ is either finite dimensional or if $X$ has only a finite number of non-zero homotopy groups, then the answer to the question is yes! However, in general, there are examples, composed by Adams [1] and Gray 4, demonstrating that the answer to this question is no!

As usual, let $\Sigma$ and $\Omega$ be the suspension and loop functors in the (pointed) homotopy category respectively. Let $\operatorname{Aut}(X)$ be a group of self-homotopy equivalences of $X$, and let $S N T(X)$ be the set of all homotopy types $[Y]$ such that $X^{(n)}$ and $Y^{(n)}$ are homotopy equivalent for each integer $n$. We can find valuable results regarding the same homotopy $n$-type and those kinds of notions (see [5, [9], 12]). In particular, Wilkerson [15, Theorem I] proved that for a connected $C W$-complex $X$, there is a bijection of pointed sets $S N T(X) \approx \lim ^{1}\left\{\operatorname{Aut}\left(X^{(n)}\right)\right\}$, where $\lim ^{1}(-)$ is the first derived limit of groups in the sense of Bousfield-Kan [2, p. 251].

The most interesting problems of the same homotopy $n$-type are the suspensions of the Eilenberg-Mac Lane spaces or the localization of these spaces at any set of primes $J[6]$. In the case of odd integers $2 d+1, d \geq 0$, it is well known that

Received by the editors February 28, 2008, and, in revised form, April 28, 2008.

2000 Mathematics Subject Classification. Primary 55P15; Secondary 55S37, 55P40.

Key words and phrases. Same $n$-type, Aut, commutator, Samelson (Whitehead) product.

This paper was (partially) supported by the Chonbuk National University funds for overseas research, 2008.

(C)2008 American Mathematical Society Reverts to public domain 28 years from publication 
$S N T\left(\Sigma^{k} K(\mathbb{Z}, 2 d+1)\right)=*$ for $k \geq 0$. The proof of this depends on the fact that the space $\Sigma^{k} K(\mathbb{Z}, 2 d+1)$ has the rational homotopy type of a single sphere in dimension $d=k+2 d+1$. What will happen in the case of even integers? As the reader can see, this is too complicated and intractable, because the space $\Sigma^{k} K(\mathbb{Z}, 2 d)$ has the rational homotopy type of a bouquet of spheres in dimensions $d=k+2 d, k+4 d, k+6 d, \cdots$. From this point of view, there is a conjecture [11, p. 287] posed by C. A. McGibbon and J. M. Møller:

Conjecture. $\operatorname{SNT}\left(\Sigma \mathbb{C} P^{\infty}\right)=*$.

The main purpose of this paper is to present the positive answer to the above conjecture. In Section 2, we describe self-maps on the suspension and loop structures and create useful self-maps of commutators on the suspension structures. In Section 3, we give the answer to the conjecture.

\section{Construction of SelF-Maps}

McGibbon and Møller [11, Theorem 1] proved

Theorem 1. Let $X$ be a 1-connected space with finite type over some subring of the rationals. Assume that $X$ has the rational homotopy type of a bouquet of spheres. Then the following three conditions are equivalent:

(1) $\operatorname{SNT}(X)=*$,

(2) the map $\operatorname{Aut}(X) \stackrel{f \mapsto f^{(n)}}{\longrightarrow} \operatorname{Aut}\left(X^{(n)}\right)$ has a finite cokernel for each $n$,

(3) the map $\operatorname{Aut}(X) \stackrel{f \mapsto f_{\sharp}}{\longrightarrow} A u t\left(\pi_{\leq n}(X)\right)$ has a finite cokernel for each $n$.

Here, the group Aut $\left(\pi_{\leq n}(X)\right)$ denotes the group of automorphisms of the graded $\mathbb{Z}$-module, $\pi_{\leq n}(X)$, preserving the Whitehead product pairings.

Actually, we will use this theorem in order to provide the answer to the conjecture. Theorem 1 is the Eckmann-Hilton dual of the corresponding result [10, Theorem 3] concerning the 1-connected $H_{0}$-space with finite type over $Z_{J}$ for some set of primes $J$ along with cohomology.

Let $\hat{\varphi}_{1}: \mathbb{C} P^{\infty} \rightarrow \Omega \Sigma \mathbb{C} P^{\infty}$ and $x_{1}: S^{2} \rightarrow \Omega \Sigma \mathbb{C} P^{\infty}$ be the inclusions. Morisugi 13] considered the maps inductively:

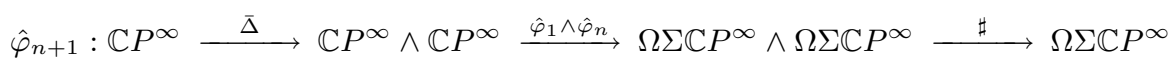

and

$$
x_{n+1}: S^{2 n+2}=S^{2} \wedge S^{2 n} \stackrel{x_{1} \wedge x_{n}}{\longrightarrow} \Omega \Sigma \mathbb{C} P^{\infty} \wedge \Omega \Sigma \mathbb{C} P^{\infty} \stackrel{\sharp}{\longrightarrow} \Omega \Sigma \mathbb{C} P^{\infty},
$$

where $\bar{\Delta}$ is the reduced diagonal map and $\sharp$ means an extension of the adjoint of the Hopf construction of $\mathbb{C} P^{\infty}$. Now we take a self-map $\varphi_{n}: \Sigma \mathbb{C} P^{\infty} \rightarrow \Sigma \mathbb{C} P^{\infty}$ by the adjoint of $\hat{\varphi}_{n}: \mathbb{C} P^{\infty} \rightarrow \Omega \Sigma \mathbb{C} P^{\infty}$ for $n=1,2, \cdots$. On the other hand, one can find a self-map $\psi^{q}: \mathbb{C} P^{\infty} \rightarrow \mathbb{C} P^{\infty}$ which corresponds to $q \in \mathbb{Z} \cong H^{2}\left(\mathbb{C} P^{\infty} ; \mathbb{Z}\right) \cong$ $\left[\mathbb{C} P^{\infty}, \mathbb{C} P^{\infty}\right]$. The suspension of this map gives a self-map $\Sigma \psi^{q}$ of $\Sigma \mathbb{C} P^{\infty}$. McGibbon [8, Theorem 1] showed that every self-map of $\Sigma \mathbb{C} P^{\infty}$ is a linear combination of these maps, $\Sigma \psi^{1}, \Sigma \psi^{2}, \cdots, \Sigma \psi^{n}$, up to homology. Indeed, we can construct self-maps of $\Sigma \mathbb{C} P^{\infty}$ by using the suspension structure, up to homology, as follows: $\varphi_{1}=\Sigma \psi^{1}, \varphi_{2}=\Sigma \psi^{2}-2 \Sigma \psi^{1}, \varphi_{3}=\Sigma \psi^{3}-3 \Sigma \psi^{2}+3 \Sigma \psi^{1}$, and in general $\varphi_{n}=\sum_{r=0}^{n-1}(-1)^{r}\left(\begin{array}{l}n \\ r\end{array}\right) \sum \psi^{n-r}$, where $\left(\begin{array}{l}n \\ r\end{array}\right)$ is a binomial coefficient. We note that these self-maps are exactly the same as the above self-maps (see [13, Theorem 1.7]) 
and $x_{n}$ is the rationally non-trivial indecomposable generator of $\pi_{2 n}\left(\Omega \Sigma \mathbb{C} P^{\infty}\right)$ for each $n$. By Theorem 1.4, ibid., we have

Theorem 2. $\left\{\hat{\varphi}_{n}\right\}$ and $\left\{x_{n}\right\}$ have the following properties:

(1) $\hat{\varphi}_{n}: \mathbb{C} P^{\infty} \rightarrow \Omega \Sigma \mathbb{C} P^{\infty}$ factors as $\mathbb{C} P^{\infty} \stackrel{p}{\rightarrow} \mathbb{C} P^{\infty} / \mathbb{C} P^{n-1} \stackrel{g_{n}}{\rightarrow} \Omega \Sigma \mathbb{C} P^{\infty}$ (where $p$ is the projection) such that the restriction to the bottom sphere of the map $g_{n}$ coincides with the map $x_{n}: S^{2 n} \rightarrow \Omega \Sigma \mathbb{C} P^{\infty}$.

(2) Let $\left[\hat{\varphi}_{m}, \hat{\varphi}_{n}\right]$ be a commutator in the group $\left[\mathbb{C} P^{\infty}, \Omega \Sigma \mathbb{C} P^{\infty}\right]$. Then $i^{*}\left[\hat{\varphi}_{m}, \hat{\varphi}_{n}\right]$ $=q^{*}<x_{m}, x_{n}>$. Here $i: \mathbb{C} P^{m+n} \rightarrow \mathbb{C} P^{\infty}$ is the inclusion, $q: \mathbb{C} P^{m+n} \rightarrow$ $S^{2 m+2 n}$ is the projection and $<x_{m}, x_{n}>$ is the Samelson product in $\pi_{*}\left(\Omega \Sigma \mathbb{C} P^{\infty}\right)$.

Similarly, let $\left[\varphi_{m}, \varphi_{n}\right]$ be the commutator of $\varphi_{m}$ and $\varphi_{n}$ in the group $\left[\Sigma \mathbb{C} P^{\infty}\right.$, $\left.\Sigma \mathbb{C} P^{\infty}\right]$. That is, $\left[\varphi_{m}, \varphi_{n}\right]=\varphi_{m}+\varphi_{n}-\varphi_{m}-\varphi_{n}$, where + and - mean the operations of maps induced by the suspension structure on $\Sigma \mathbb{C} P^{\infty}$. We note that the restriction $\left.\left[\varphi_{m}, \varphi_{n}\right]\right|_{\left(\Sigma \mathbb{C} P{ }^{\infty}\right)_{2 m+2 n}}$ to the skeleton is inessential (see [7]).

Remark 3. In order to produce enough self-maps in $\operatorname{Aut}\left(\Sigma \mathbb{C} P^{\infty}\right)$ so that the map $\operatorname{Aut}\left(\Sigma \mathbb{C} P^{\infty}\right) \rightarrow \operatorname{Aut}\left(\pi_{\leq n}\left(\Sigma \mathbb{C} P^{\infty}\right)\right)$ has a finite cokernel for each $n$, we now construct the self-homotopy equivalences of $\Sigma \mathbb{C} P^{\infty}$ by the form $I+\left[\varphi_{i_{n}},\left[\varphi_{i_{n-1}}, \cdots\right.\right.$, $\left.\left.\left[\varphi_{i_{1}}, \varphi_{i_{2}}\right], \cdots\right]\right]$, where $I$ is the identity map on $\Sigma \mathbb{C} P^{\infty}$ and $\left[\varphi_{i_{n}},\left[\varphi_{i_{n-1}}, \cdots,\left[\varphi_{i_{1}}, \varphi_{i_{2}}\right]\right.\right.$, ...]] is the iterated commutator of self-maps $\varphi_{i_{j}}$.

We recall that the Samelson product gives $\pi_{*}(\Omega X), * \geq 1$, the structure of graded Lie algebra ([14, and [3, p. 141); that is, $\langle x, y\rangle=-(-1)^{|x||y|}\langle y, x\rangle$, and $\left\langle x,<y, z>>=<<x, y>, z>+(-1)^{|x||y|}<y,<x, z>>\right.$. For another notion in order to understand the given conjecture, we require a steady calculation in $\pi_{*}\left(\Omega \Sigma \mathbb{C} P^{\infty}\right)\left(\right.$ or $\left.\pi_{*}\left(\Sigma \mathbb{C} P^{\infty}\right)\right)$. Let $L$ and $L_{\leq n}$ denote the Lie algebras defined by the Samelson products on the quotients $\pi_{*}\left(\Omega \Sigma \mathbb{C} P^{\infty}\right) /$ torsion and $\pi_{\leq 2 n}\left(\Omega \Sigma \mathbb{C} P^{\infty}\right) /$ torsion, respectively. That is,

$$
L=\pi_{*}\left(\Omega \Sigma \mathbb{C} P^{\infty}\right) / \text { torsion }\left(=L<x_{1}, x_{2}, \cdots, x_{n}, \cdots>, \operatorname{dim} x_{n}=2 n\right)
$$

and

$$
L_{\leq n}=\pi_{\leq 2 n}\left(\Omega \Sigma \mathbb{C} P^{\infty}\right) / \text { torsion }=\pi_{*}\left(\Omega \Sigma \mathbb{C} P^{\infty}\right)^{(2 n)} / \text { torsion },
$$

where $\left(\Omega \Sigma \mathbb{C} P^{\infty}\right)^{(2 n)}$ means the Postnikov approximation and $x_{n}: S^{2 n} \rightarrow \Omega \Sigma \mathbb{C} P^{\infty}$ is the rationally non-trivial indecomposable generator in $\pi_{2 n}\left(\Omega \Sigma \mathbb{C} P^{\infty}\right), n=1,2$, $3, \cdots$, as mentioned earlier. Equivalently, under the same letter,

$$
L=\pi_{*}\left(\Sigma \mathbb{C} P^{\infty}\right) / \text { torsion }\left(=L<x_{1}, x_{2}, \cdots, x_{n}, \cdots>\right),
$$

where $x_{n}$ is also the rationally non-trivial indecomposable generator in $\pi_{2 n+1}\left(\Sigma \mathbb{C} P^{\infty}\right)$, and the operation in this case is the Whitehead product on $\pi_{*}\left(\Sigma \mathbb{C} P^{\infty}\right) /$ torsion. We note that the torsion generators can be ignored in the SNTcomputations. Throughout this paper we write the rationally non-trivial indecomposable generators in $\pi_{\leq 2 n}\left(\Omega \Sigma \mathbb{C} P^{\infty}\right)$ /torsion by the same letter $\left(x_{1}, x_{2}, \cdots, x_{n}, \cdots\right)$ in $\pi_{\leq 2 n+1}\left(\Sigma \mathbb{C} P^{\infty}\right) /$ torsion for each $n$.

We recall that $\tilde{H}_{*}\left(\mathbb{C} P^{\infty} ; \mathbb{Z}\right) \cong \mathbb{Z}\left\{b_{1}, b_{2}, \cdots, b_{n}, \cdots\right\}$ as a $\mathbb{Z}$-module, where $b_{n} \in$ $H_{2 n}\left(\mathbb{C} P^{\infty} ; \mathbb{Z}\right)$ is the standard generator. The rational homology of $\Omega \Sigma \mathbb{C} P^{\infty}$ is a tensor algebra $T<b_{1}, b_{2}, \cdots, b_{n}, \cdots>$ generated by $\left\{b_{1}, b_{2}, \cdots, b_{n}, \cdots\right\}$, where $b_{n}$ is a generator of $H_{2 n}\left(\Omega \Sigma \mathbb{C} P^{\infty} ; \mathbb{Q}\right)$ with diagonal $\Delta\left(b_{k}\right)=\sum_{i+j=k} b_{i} \otimes b_{j}$ and $b_{n}=E_{*}\left(b_{n}\right)$. Here, $E$ is the inclusion map $E: \mathbb{C} P^{\infty} \rightarrow \Omega \Sigma \mathbb{C} P^{\infty}$ defined by $E(x)(t)=(x, t) \in \Sigma \mathbb{C} P^{\infty}$, and $b_{0}$ means $1 \in H_{0}\left(\Omega \Sigma \mathbb{C} P^{\infty}\right)$. 
Let $h: \pi_{*}\left(\Omega \Sigma \mathbb{C} P^{\infty}\right) \rightarrow H_{*}\left(\Omega \Sigma \mathbb{C} P^{\infty}\right)$ be the Hurewicz homomorphism and let $\chi: \Omega \Sigma \mathbb{C} P^{\infty} \rightarrow \Omega \Sigma \mathbb{C} P^{\infty}$ be the map of loop inverse. Then, in [13] we have

$h\left(x_{n}\right)= \begin{cases}b_{1} & \text { if } n=1, \\ (n-1) ! \sum_{i=1}^{n} \chi_{*}\left(b_{n-i}\right)\left(i b_{i}-b_{1} b_{i-1}\right)=n ! b_{n}+\text { decomposables } & \text { if } n \geq 2,\end{cases}$

where the product in the above equations is the one in the tensor algebra.

\section{Proof of the conjecture}

From now on, let $P$ denote the infinite complex projective space $\mathbb{C} P^{\infty}$ for brevity.

Proof. Let $A_{n}=\operatorname{Aut}\left(\pi_{\leq 2 n+1}(\Sigma P) /\right.$ torsion $)$. Then we can verify that $A_{1}=\operatorname{Aut}(\mathbb{Z})$ $\cong\{ \pm 1\} \cong \mathbb{Z}_{2}$, and $A_{2} \cong\{ \pm 1\} \oplus\{ \pm 1\} \cong \mathbb{Z}_{2} \oplus \mathbb{Z}_{2}$, and that $A_{n}(n \geq 3)$ has a different group structure which is infinite (see [7). Note that if $\gamma_{0} \in \pi_{m}(\Omega X)$ and $\gamma_{1} \in \pi_{n}(\Omega X)$, then $\left[h\left(\gamma_{0}\right), h\left(\gamma_{1}\right)\right]=h\left(<\gamma_{0}, \gamma_{1}>\right)$. Let $\left[\hat{\varphi}_{m}, \hat{\varphi}_{n}\right]=\left[\widehat{\varphi_{m}, \varphi_{n}}\right]: P \rightarrow$ $\Omega \Sigma P$ be the adjoint of $\left[\varphi_{m}, \varphi_{n}\right]: \Sigma P \rightarrow \Sigma P$. Then we have

Proposition 3.1. $\left[\hat{\varphi_{m}}, \hat{\varphi_{n}}\right]_{*}\left(b_{m+n}\right)=\left[h\left(x_{m}\right), h\left(x_{n}\right)\right]$, where $b_{m+n}$ is a generator of $H_{2 m+2 n}(P),\left[h\left(x_{m}\right), h\left(x_{n}\right)\right]=h\left(x_{m}\right) h\left(x_{n}\right)-(-1)^{\left|h\left(x_{m}\right)\right|\left|h\left(x_{n}\right)\right|} h\left(x_{n}\right) h\left(x_{m}\right)$, and $x_{m}$ and $x_{n}$ are rationally non-trivial indecomposable generators.

In the particular case, we are able to see that $\left[\hat{\varphi}_{1}, \hat{\varphi}_{2}\right]_{*}\left(b_{3}\right)=2\left(b_{1} b_{2}-b_{2} b_{1}\right)=$ $\left[b_{1}, 2 b_{2}-b_{1}^{2}\right]=\left[h\left(x_{1}\right), h\left(x_{2}\right)\right]$. The following is the general case:

Proof. Let $q: \mathbb{C} P^{m+n} \rightarrow S^{2 m+2 n}$ be the projection map and let $<,>$ be the Samelson product in $\pi_{*}(\Omega \Sigma P)$. Theorem 2 says that the following diagram is commutative:

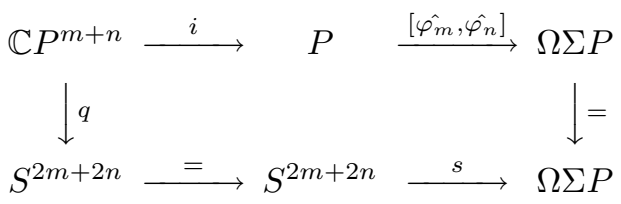

where $s=<x_{m}, x_{n}>$. Considering $\mathbb{C} P^{m+n-1} \hookrightarrow \mathbb{C} P^{m+n} \stackrel{q}{\rightarrow} \mathbb{C} P^{m+n} / \mathbb{C} P^{m+n-1}$ $=S^{2 m+2 n}$, and applying the homology to the above diagram, we have

$$
\begin{aligned}
{\left[\hat{\varphi_{m}}, \hat{\varphi_{n}}\right]_{*}\left(b_{m+n}\right) } & =\left[\hat{\varphi_{m}}, \hat{\varphi_{n}}\right]_{*} i_{*}\left(b_{m+n}\right)=s_{*} q_{*}\left(b_{m+n}\right) \\
& =s_{*}\left(b_{m+n}^{\prime}\right)=h\left(<x_{m}, x_{n}>\right) \text { (Hurewicz homomorphism) } \\
& =\left[h\left(x_{m}\right), h\left(x_{n}\right)\right] .
\end{aligned}
$$

Here, $b_{m+n}$ is also used as a generator of $H_{2 m+2 n}\left(\mathbb{C} P^{m+n}\right)\left(\cong H_{2 m+2 n}(P), i_{*}\left(b_{m+n}\right)\right.$ $\left.=b_{m+n}\right), q_{*}: H_{2 m+2 n}\left(\mathbb{C} P^{m+n}\right) \rightarrow H_{2 m+2 n}\left(S^{2 m+2 n}\right)$ is an isomorphism (by using a homology sequence) sending the generator $b_{m+n}$ to the fundamental homology class $b_{m+n}^{\prime}$, and $x_{m}$ and $x_{n}$ are rationally non-trivial indecomposable generators.

What will happen in the homomorphism induced by the self-maps $I+\left[\varphi_{m}, \varphi_{n}\right]$ in the homotopy groups? The answer to this query can be found as follows:

Lemma 3.2. Let $p=2 m+2 n+1$, and let $x_{m+n}$ be the indecomposable generator in $\pi_{p}(\Sigma P) /$ torsion. Then $\left(I+\left[\varphi_{m}, \varphi_{n}\right]\right)_{\sharp}\left(x_{m+n}\right)=x_{m+n}+\left[\varphi_{m}, \varphi_{n}\right]_{\sharp}\left(x_{m+n}\right)$, where the first + is the addition of maps induced by the suspension structure on $\Sigma P$, while the second + refers to the one of homotopy groups, and $f_{\sharp}$ denotes an induced homomorphism in homotopy groups. 
Proof. We must show that the diagram

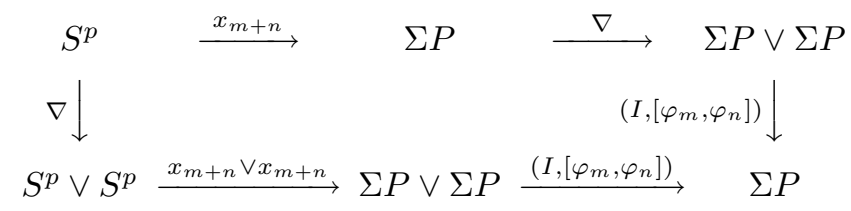

is commutative. Consider the following diagram:

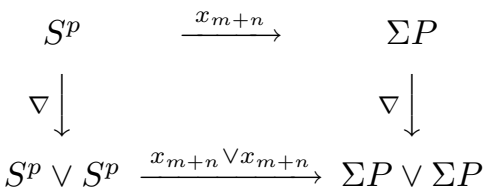

Note that the diagram is not commutative, because $x_{m+n}$ is not a suspension. However, if we consider a quotient map $q: \Sigma P \rightarrow \Sigma P /(\Sigma P)_{p-1}$, then the following two diagrams are commutative:

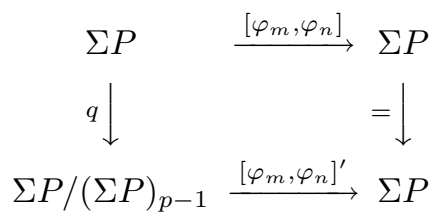

and

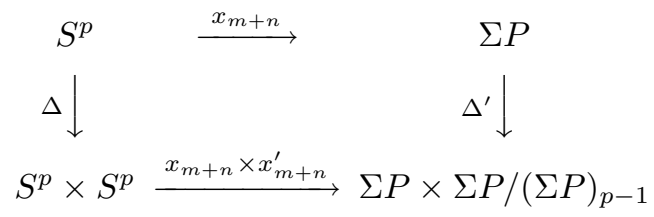

where $\left[\varphi_{m}, \varphi_{n}\right]^{\prime}$ is a map induced by the commutator $\left[\varphi_{m}, \varphi_{n}\right]$ making the above diagram commutative (this map could be guaranteed since the restriction $\left.\left[\varphi_{m}, \varphi_{n}\right]\right|_{(\Sigma P)_{2 m+2 n}}$ to the skeleton is inessential), and $\Delta(x)=(x, x), \Delta^{\prime}(x)=$ $(x, q(x))$ and $x_{m+n}^{\prime}=q\left(x_{m+n}\right)$. Also note that since $\Sigma P /(\Sigma P)_{p-1}$ is $p-1$ connected,

$$
\left[S^{p}, \Sigma P \vee \Sigma P /(\Sigma P)_{p-1}\right] \cong\left[S^{p}, \Sigma P \times \Sigma P /(\Sigma P)_{p-1}\right] .
$$

This isomorphism takes $\left(x_{m+n} \vee x_{m+n}^{\prime}\right) \circ \nabla \mapsto\left(x_{m+n} \times x_{m+n}^{\prime}\right) \circ \Delta$, and it also takes $\Delta^{\prime}\left(x_{m+n}\right)$ to $\nabla^{\prime}\left(x_{m+n}\right)$. Therefore the diagram

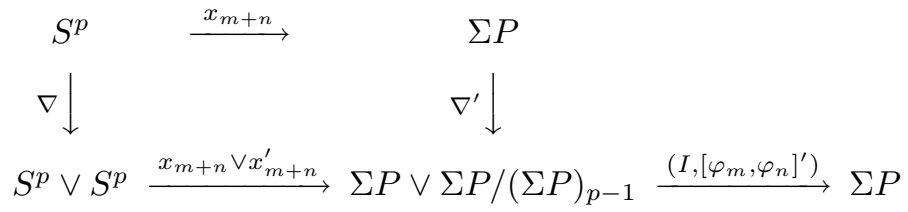

commutes. Thus we complete the proof.

It is not difficult to show that the above lemma still holds for the iterated commutators from the fact that $\left.\left[\varphi_{i_{k}},\left[\varphi_{i_{k-1}}, \cdots,\left[\varphi_{i_{1}}, \varphi_{i_{2}}\right], \cdots\right]\right]\right|_{(\Sigma P)_{2\left(i_{1}+i_{2}+\cdots+i_{k}\right)}} \simeq *$. Moreover, it can be seen that there are many rationally non-trivial indecomposable and decomposable generators on $\pi_{2 n+1}(\Sigma P) \otimes \mathbb{Q}\left(\right.$ or $\left.\pi_{2 n}(\Omega \Sigma P) \otimes \mathbb{Q}\right)$. For example, $\left\{x_{1}\right\}$ in dimension $3,\left\{x_{2}\right\}$ in dimension $5,\left\{x_{3},\left[x_{1}, x_{2}\right]\right\}$ in dimension 7 , $\left\{x_{4},\left[x_{1}, x_{3}\right],\left[x_{1},\left[x_{1}, x_{2}\right]\right]\right\}$ in dimension $9,\left\{x_{5},\left[x_{1}, x_{4}\right],\left[x_{1},\left[x_{1}, x_{3}\right]\right],\left[x_{1},\left[x_{1}\left[x_{1}, x_{2}\right]\right]\right]\right.$, $\left.\left[x_{2}, x_{3}\right],\left[x_{2},\left[x_{1}, x_{2}\right]\right]\right\}$ in dimension 11 , and so on. Indeed, we can show that the 
above (iterated) Whitehead products are rationally non-trivial by using the cohomology cup products.

Lemma 3.3. For each Whitehead product $\left[x_{i_{k}},\left[x_{i_{k-1}}, \cdots,\left[x_{i_{1}}, x_{i_{2}}\right], \cdots\right]\right]$ in $\pi_{*}(\Sigma P)$, there exists an iterated commutator $\left[\varphi_{i_{k}},\left[\varphi_{i_{k-1}}, \cdots,\left[\varphi_{i_{1}}, \varphi_{i_{2}}\right], \cdots\right]\right]$ in the group $[\Sigma P, \Sigma P]$ such that

$\left(I+\left[\varphi_{i_{k}},\left[\varphi_{i_{k-1}}, \cdots,\left[\varphi_{i_{1}}, \varphi_{i_{2}}\right], \cdots\right]\right]\right)_{\sharp}\left(x_{n}\right)=x_{n}+n !\left[x_{i_{k}},\left[x_{i_{k-1}}, \cdots,\left[x_{i_{1}}, x_{i_{2}}\right], \cdots\right]\right]$, where $x_{n}$ and $x_{i_{j}}$ are rationally non-trivial indecomposable generators and $n=$ $i_{1}+i_{2}+\cdots+i_{k}$.

Remark 3.4. We note that the phenomena of the iterated commutators completely depend on those of iterated Whitehead products. What is even more interesting is that the map $\left[\varphi_{i_{k}},\left[\varphi_{i_{k-1}}, \cdots,\left[\varphi_{i_{1}}, \varphi_{i_{2}}\right], \cdots\right]\right]_{\sharp}$ sends the indecomposable generators to the (iterated) Whitehead products which are rationally non-trivial decomposable. This fact is just what we need in the proof of the conjecture!

Proof of Lemma 3.3. In the case of the one-fold Whitehead product, we need to show that $\left[\varphi_{i_{1}}, \varphi_{i_{2}}\right]_{\sharp}\left(x_{n}\right)=n !\left[x_{i_{1}}, x_{i_{2}}\right]\left(n=i_{1}+i_{2}\right)$. The proof follows by chasing the diagram in Proposition 3.1 (by adjointness) and the following commutative diagram:

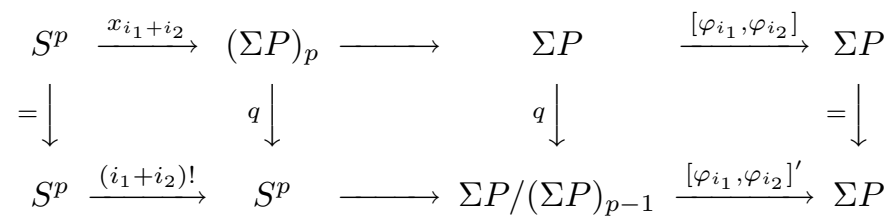

where $p=2 n+1, n=i_{1}+i_{2}$, and the $q$ 's are the projections.

We suppose that for the iterated Whitehead products $\left[x_{i_{k-1}}, \cdots,\left[x_{i_{1}}, x_{i_{2}}\right], \cdots\right]$ of length greater than 2 , there exist iterated commutators $\left[\varphi_{i_{k-1}}, \cdots,\left[\varphi_{i_{1}}, \varphi_{i_{2}}\right], \cdots\right]$ such that

$$
\left(I+\left[\varphi_{i_{k-1}}, \cdots,\left[\varphi_{i_{1}}, \varphi_{i_{2}}\right], \cdots\right]\right)_{\sharp}\left(x_{m}\right)=x_{m}+m !\left[x_{i_{k-1}}, \cdots,\left[x_{i_{1}}, x_{i_{2}}\right], \cdots\right],
$$

where $x_{m}$ and $x_{i_{j}}$ are rationally non-trivial indecomposable generators, and $m=$ $i_{1}+i_{2}+\cdots+i_{k-1}$. We recall that the restriction $\left.\left[\varphi_{i_{k-1}}, \cdots,\left[\varphi_{i_{1}}, \varphi_{i_{2}}\right], \cdots\right]\right|_{(\Sigma P)_{2 m}}$ to the skeleton is trivial in homotopy and the map $\left[x_{i_{k-1}}, \cdots,\left[x_{i_{1}}, x_{i_{2}}\right], \cdots\right]: S^{2 m+1} \rightarrow$ $\Sigma P$ is a rationally non-trivial decomposable generator (when rationalized). By applying the first statement and the above results, we can find an iterated commutator $\left[\varphi_{i_{k}},\left[\varphi_{i_{k-1}}, \cdots,\left[\varphi_{i_{1}}, x_{i_{2}}\right], \cdots\right]\right]$ such that the desired formula is still guaranteed.

As previously described, $L$ and $L_{\leq n}$ denote the Lie algebras defined by the Whitehead products on $\pi_{*}(\Sigma P) /$ torsion and $\pi_{\leq 2 n+1}(\Sigma P) /$ torsion (or equivalently the Samelson products on the quotients $\pi_{*}(\Omega \Sigma P) /$ torsion and $\pi_{\leq 2 n}(\Omega \Sigma P) /$ torsion) respectively. We note that $\Sigma P$ has the rational homotopy type of the bouquet of spheres, i.e., $\Sigma P \simeq_{0} S^{3} \vee S^{5} \vee S^{7} \vee \cdots$, where $\simeq_{0}$ means a rational homotopy. Following McGibbon and Møller [11, we can construct a short exact sequence

$$
0 \rightarrow \operatorname{Hom}\left(Q_{n} L, D_{n} L\right) \rightarrow \operatorname{Aut}\left(L_{\leq n}\right) \rightarrow \operatorname{Aut}\left(L_{<n}\right) \oplus \operatorname{Aut}\left(Q_{n} L\right) \rightarrow 0 .
$$

Here, $Q_{n} L$ and $D_{n} L$ are indecomposables and decomposables respectively, and the maps are given in the same method (see below). Since $\operatorname{Aut}(T)$ is finite, where $T$ is torsion in $\pi_{\leq 2 n+1}(\Sigma P)$, in order to prove the given conjecture it suffices to show that the map $\operatorname{Aut}(\Sigma P) \rightarrow \operatorname{Aut}\left(L_{\leq n}\right)$ has a finite cokernel for each $n$. Using 
the previous results of this paper, we prove the conjecture by the induction step on $n$. Since $A_{1}=\operatorname{Aut}\left(L_{\leq 1}\right) \cong \operatorname{Aut}(\mathbb{Z}) \cong \mathbb{Z}_{2}$, the initial step is trivial. Suppose that the map $\operatorname{Aut}(\Sigma P) \rightarrow \operatorname{Aut}\left(L_{<n}\right)$ has a finite cokernel. Let $\mathcal{D}_{n}$ be the set of (iterated) Whitehead products in $\pi_{2 n+1}(\Sigma P) /$ torsion which are, after rationalized, decomposable generators in $\pi_{2 n+1}(\Sigma P) \otimes \mathbb{Q}$ for each $n \geq 3$ (say, $\mathcal{D}_{3}=\left\{\left[x_{1}, x_{2}\right]\right\}$, $\mathcal{D}_{4}=\left\{\left[x_{1}, x_{3}\right],\left[x_{1},\left[x_{1}, x_{2}\right]\right]\right\}, \mathcal{D}_{5}=\left\{\left[x_{1}, x_{4}\right],\left[x_{1},\left[x_{1}, x_{3}\right]\right],\left[x_{1},\left[x_{1}\left[x_{1}, x_{2}\right]\right]\right],\left[x_{2}, x_{3}\right]\right.$, $\left.\left[x_{2}, \quad\left[x_{1}, x_{2}\right]\right]\right\}$, and so on). Then, for each (iterated) Whitehead product $\left[x_{i_{k}},\left[x_{i_{k-1}}, \cdots,\left[x_{i_{1}}, x_{i_{2}}\right], \cdots\right]\right] \in \mathcal{D}_{n}\left(n=i_{1}+i_{2}+\cdots+i_{k}\right)$, by Lemmas 3.2 and 3.3 , we can produce a self-map $I+\left[\varphi_{i_{k}},\left[\varphi_{i_{k-1}}, \cdots,\left[\varphi_{i_{1}}, \varphi_{i_{2}}\right], \cdots\right]\right] \in \operatorname{Aut}(\Sigma P)$ such that the restriction $\left(I+\left.\left[\varphi_{i_{k}},\left[\varphi_{i_{k-1}}, \cdots,\left[\varphi_{i_{1}}, \varphi_{i_{2}}\right], \cdots\right]\right)_{\sharp}\right|_{L_{<n}}\right.$ is the identity, and

$\left(I+\left[\varphi_{i_{k}},\left[\varphi_{i_{k-1}}, \cdots,\left[\varphi_{i_{1}}, \varphi_{i_{2}}\right], \cdots\right]\right]\right)_{\sharp}\left(x_{n}\right)=x_{n}+n !\left[x_{i_{k}},\left[x_{i_{k-1}}, \cdots,\left[x_{i_{1}}, x_{i_{2}}\right], \cdots\right]\right]$, where $x_{n}$ is a rationally non-trivial indecomposable generator in $\pi_{2 n+1}(\Sigma P) /$ torsion. We now consider the diagram

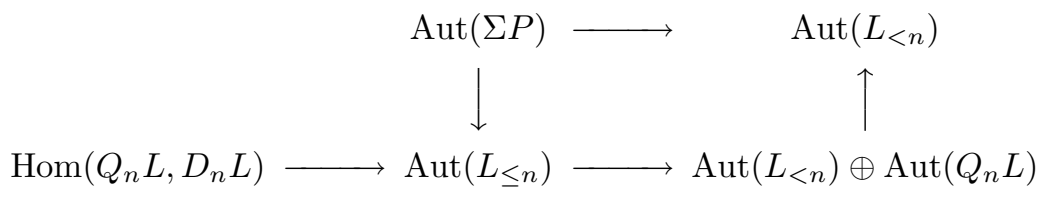

and the composition

$$
L_{\leq n} \stackrel{q}{\longrightarrow} Q_{n} L \stackrel{\left[\varphi_{i_{k}},\left[\varphi_{i_{k-1}}, \cdots,\left[\varphi_{i_{1}}, \varphi_{i_{2}}\right], \cdots\right]\right]_{\sharp}}{\longrightarrow} D_{n} L \stackrel{j}{\longrightarrow} L_{\leq n}
$$

of maps. Here,

(1) $q$ is the projection and $j$ is the inclusion;

$(2)$ the map $\operatorname{Hom}\left(Q_{n} L, D_{n} L\right) \rightarrow \operatorname{Aut}\left(L_{\leq n}\right)$ sends $\left[\varphi_{i_{k}},\left[\varphi_{i_{k-1}}, \cdots,\left[\varphi_{i_{1}}, \varphi_{i_{2}}\right]\right.\right.$, $\cdots]]_{\sharp}$ to $I+j \circ\left[\varphi_{i_{k}},\left[\varphi_{i_{k-1}}, \cdots,\left[\varphi_{i_{1}}, \varphi_{i_{2}}\right], \cdots\right]\right]_{\sharp} \circ q$, and the map out of $\operatorname{Aut}\left(L_{\leq n}\right)$ is given by restriction and projection; and

(3) the right vertical arrow is the projection.

Note that the (iterated) commutators $\left[\varphi_{i_{k}},\left[\varphi_{i_{k-1}}, \cdots,\left[\varphi_{i_{1}}, \varphi_{i_{2}}\right], \cdots\right]\right]$ completely depend on the types of (iterated) Whitehead products $\left[x_{i_{k}},\left[x_{i_{k-1}}, \cdots,\left[x_{i_{1}}, x_{i_{2}}\right], \cdots\right]\right]$ as in Lemma 3.3. We also note that the map $\operatorname{Aut}\left(L_{\leq n}\right) \rightarrow \operatorname{Aut}\left(L_{<n}\right) \oplus \operatorname{Aut}\left(Q_{n} L\right)$ is an epimorphism and that $\operatorname{Aut}\left(Q_{n} L\right) \cong \operatorname{Aut}(\mathbb{Z}) \cong \mathbb{Z}_{2}$ because $Q_{n} L$ has the only one indecomposable generator, up to sign, for each $n$. Those facts and the induction hypothesis force the map $\operatorname{Aut}(\Sigma P) \rightarrow \operatorname{Aut}\left(L_{\leq n}\right)$ to have a finite cokernel as required.

\section{ACKNOWLEDGEMENTS}

I would like to express my gratitude to Professor C. A. McGibbon for his encouragement and fruitful discussion on this paper while I was staying at Wayne State University. The author is also grateful to the referee(s) for offering other suggestions that improved the quality of the paper.

\section{REFERENCES}

1. J. F. Adams, An example in homotopy theory, Proc. Camb. Phil. Soc. 53 (1957), 922-923. MR0091477 (19:975d)

2. A. K. Bousfield and D. M. Kan, Homotopy limits, completions and localizations, Lecture Notes in Math. 304, Springer-Verlag, Berlin-New York, 1972. MR0365573 (51:1825) 
3. F. R. Cohen, J. C. Moore and J. A. Neisendorfer, Torsion in homotopy groups, Ann. of Math. (2) 109 (1979), 121-168. MR519355 (80e:55024)

4. B. I. Gray, Spaces of the same n-type, for all n, Topology 5 (1966), 241-243. MR0196743 $(33: 4929)$

5. J. R. Harper and J. Roitberg, Phantom maps and spaces of the same $n$-type for all $n$, J. Pure Appl. Algebra 80 (1992), 123-137. MR1172722 (93g:55010)

6. P. Hilton, G. Mislin and J. Roitberg, Homotopical localization, Proc. London Math. Soc. 26 (1973), 693-706. MR0326720(48:5063)

7. D. Lee, On self-homotopy equivalences of $\Sigma \mathbb{C} P^{\infty}$, preprint.

8. C. A. McGibbon, Self-maps of projective spaces, Trans. Amer. Math. Soc. 271 (1982), 325346. MR648096 (83h:55007)

9. C. A. McGibbon and J. M. Møller, How can you tell two spaces apart when they have the same n-type for all n?, Adams Memorial Symposium on Algebraic Topology, N. Ray and G. Walker, eds., London Math. Soc. Lecture Note Series 175, Cambridge Univ. Press, Cambridge, 1992, 131-143. MR.1170575 (93i:55010)

10. C. A. McGibbon and J. M. Møller, On spaces with the same $n$-type for all $n$, Topology 31 (1992), 177-201. MR.1153244 (92m:55008)

11. C. A. McGibbon and J. M. Møller, On infinite dimensional spaces that are rationally equivalent to a bouquet of spheres, Proceedings of the 1990 Barcelona Conference on Algebraic Topology, Lecture Notes in Math. 1509, Springer, Berlin, 1992, 285-293. MR1185978 (93h:55012)

12. C. A. McGibbon and J. Roitberg, Phantom maps and rational equivalences, Amer. J. Math. 116 (1994), 1365-1379. MR1305869 (95j:55026)

13. K. Morisugi, Projective elements in K-theory and self-maps of $\Sigma \mathbb{C} P^{\infty}$, J. Math. Kyoto Univ. 38 (1998), 151-165. MR1628087 (99g:55008)

14. G. W. Whitehead, Elements of homotopy theory, GTM 61, Springer-Verlag, New YorkHeidelberg-Berlin, 1978. MR516508 (80b:55001)

15. C. W. Wilkerson, Classification of spaces of the same n-type for all n, Proc. Amer. Math. Soc. 60 (1976), 279-285. MR0474283 (57:13930)

Department of Mathematics, and Institute of Pure and Applied Mathematics, ChonBuk National University, Jeonju, Jeonbuk 561-756, Republic of Korea

E-mail address: dwlee@math.chonbuk.ac.kr 